Med Klin Intensivmed Notfmed 2021 · 116:136-137 https://doi.org/10.1007/s00063-020-00746-4 Online publiziert: 23. Oktober 2020

(c) Springer Medizin Verlag GmbH, ein Teil von Springer Nature 2020

\section{Ursachen einer Sekretretention}

- Vermehrte Sekretbildung

- Reduzierte mukoziliäre Clearance

- ${ }^{2}$ Ineffektiver Hustenstoß

- Rezidivierende Aspirationen

[6]

\section{Zeichen der Sekretansammlung}

- Patient gibt Bedarf an

- Rasselgeräusche (z. B. Auskultation)

- Abfall des AZV bei druckkontrollierter Beatmung (Anstieg des inspiratorischen Drucks bei volumenkontrollierter Beatmung)

- Sichtbare Sekrete

- Abfall der $\mathrm{O}_{2}$-Sättigung

- Radiologie

- Tubuskontrolle

- Probenentnahme

[4]

\section{${ }^{4}$ Nebenwirkungen}

- Veränderungen der Lungencompliance/Residualkapazität

- Atelektasen

- Hypoxie/Hypoxämie

- Verletzungen der Mukosa

- Mikrobielle Kolonisation in den tieferen Atemwegen

- Veränderung des zerebralen Blutflusses/Anstieg des intrakraniellen Drucks

Arnold Kaltwasser · Rolf Dubb

Akademie der Kreiskliniken Reutlingen $\mathrm{GmbH}$, Reutlingen, Deutschland

\title{
Endotracheales Absaugen
}

- Hyper-/Hypotension

- Herzrhythmusstörungen

[1]

5 Indikationen für geschlossenes Absaugsystem

- Schwere Oxygenierungsstörung

- PEEP >6-8 mbar

- Infektion (z.B. offene TB, MRSA, COVID-19)

- Immunsuppression

- Lagerungsbehandlung

- Erhöhter Hirndruck

- Hämodynamische Instabilität

[6]

\section{${ }^{6}$ Grundlagen der}

Komplikationsvermeidung

- Seitliche Öffnungen an der Katheterspitze

- Längenmarkierungen

- Innenlumen des Tubus maximal zur Hälfte verlegen

- Soghöhe 80-120 mm Hg (max. $150 \mathrm{~mm} \mathrm{Hg}$ )

- Präoxygenierung 30-60s

- Recruitment-Manöver, kritische Indikationsstellung

- ${ }^{7}$ Hygienerichtlinien/Eigenschutz beachten

- Absaugtiefe max. 0,5-1 cm über (Trachealkanülen-)Tubusende

- Absaugdauer 15-20s

- Lavage nur unter bronchoskopischer Sicht
- Bei Entblockung des Cuffs Aspiration vermeiden

- ${ }^{8}$ Indikation Palliativversorgung kritisch überprüfen

[1-5]

\section{${ }^{9}$ Dokumentation}

Menge, Farbe, Geruch, Konsistenz, Komplikationen

[4]

\section{Korrespondenzadresse}

\section{Arnold Kaltwasser}

Akademie der Kreiskliniken Reutlingen $\mathrm{GmbH}$ Steinenbergstr. 31, 72764 Reutlingen,

Deutschland

kaltwasser_a@klin-rt.de

\section{Einhaltung ethischer Richtlinien}

Interessenkonflikt. A. Kaltwasser hat unabhängig von der eingereichten Arbeit finanzielle Zuwendungen von Avanos, NOVO und ORION Pharma erhalten. R. Dubb hat unabhängig von der eingereichten Arbeit finanzielle Zuwendungen von Avanos, NOVO und ORION Pharma erhalten.

Für diesen Beitrag wurden von den Autoren keine Studien an Menschen oder Tieren durchgeführt. Für die aufgeführten Studien gelten die jeweils dort angegebenen ethischen Richtlinien.

\section{Literatur}

1. AARC (2010) Endotracheal suctioning of mechanically ventilated patients with artificial airways. Respir Care 55(6):758-764

2. Arcuri JF, Abarshi E, Preston NJ et al (2016) Benefits of interventions for respiratory secretion

Die hochgestellten Zahlen verweisen auf die entsprechenden Informationen in • Abb. 1 (Algorithmus: Endotracheales Absaugen beim erwachsenen kritisch kranken Patienten). 


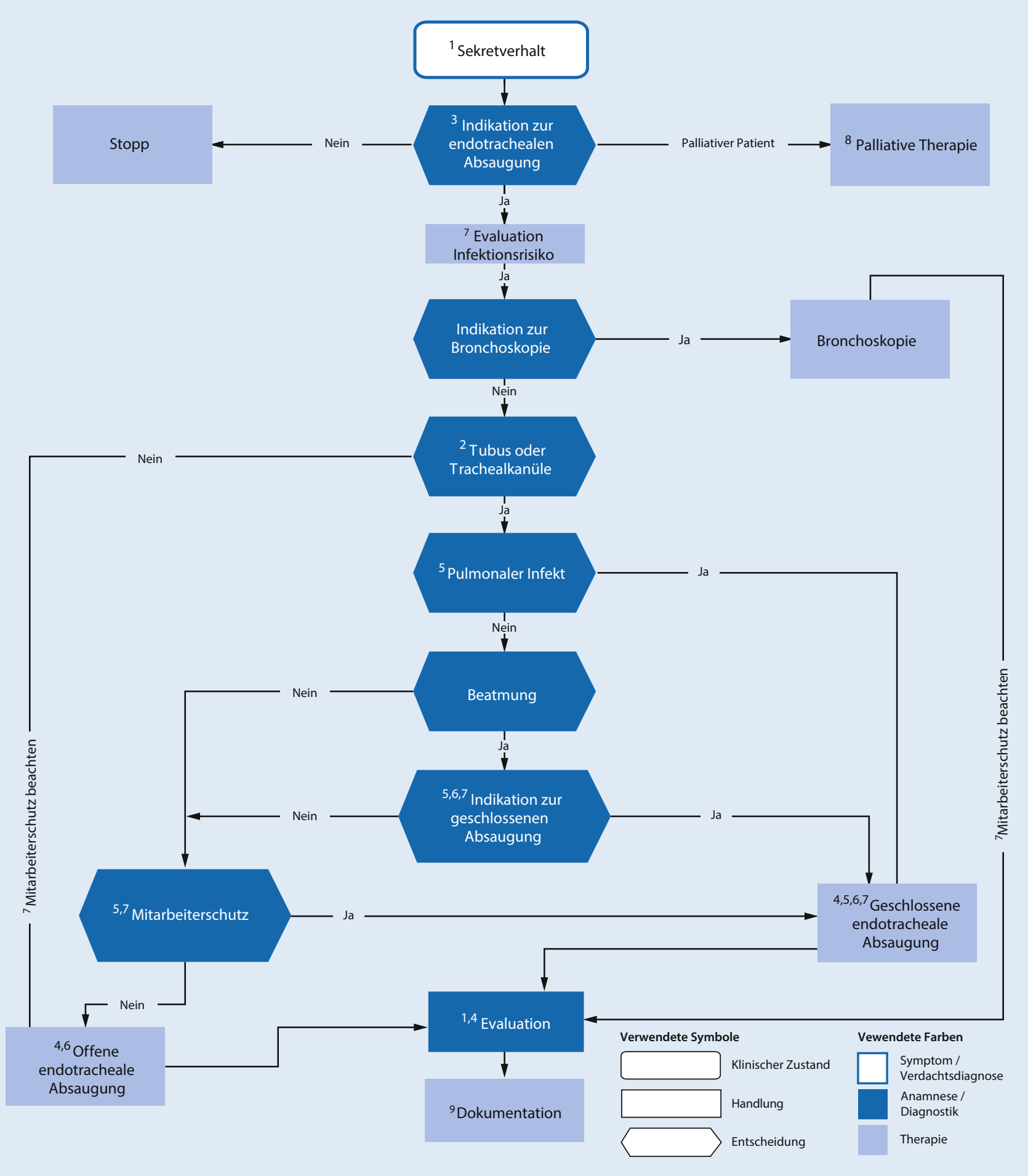

Abb. $1 \Delta$ Endotracheales Absaugen beim erwachsenen kritisch kranken Patienten

management in adult palliative care patients-a systematic review. BMC Palliat Care 15:74. https:// doi.org/10.1186/s12904-016-0147-y

3. Kaltwasser A, Dubb R, Hermes C, Rothaug $O$ (2014) Endotracheales Absaugen beim beatmeten erwachsenen Intensivpatienten: eine evidenzbasierte Checkliste. Hyg Med 39(7/8):308-311
4. Kaltwasser A, Dubb R, Rothaug O (2017) Endotracheales Absaugen: Invasiver Eingriff mit Risiken. PflegenIntensiv 17(3):26-28

5. RKI (2013) Prävention der nosokomialen beatmungsassoziierten Pneumonie. Bundesgesundheitsblatt Gesundheitsforschung Gesundheitsschutz. https://doi.org/10.1007/s00103-0131846-7
6. Schwabbauer N, Riessen R (Hrsg) (2013) Sekretmanagement in der Beatmungsmedizin, 2. Aufl. UNIMED Science, Bremen 